\title{
Motion by volume preserving mean curvature flow near cylinders
}

\author{
DAVID HARTLEY
}

\begin{abstract}
We investigate the volume preserving mean curvature flow with Neumann boundary condition for hypersurfaces that are graphs over a cylinder. Through a center manifold analysis we find that initial hypersurfaces sufficiently close to a cylinder of large enough radius, have a flow that exists for all time and converges exponentially fast to a cylinder. In particular, we show that there exist global solutions to the flow that converge to a cylinder, which are initially non-axially symmetric. A similar case where the initial hypersurfaces are spherical graphs has previously been investigated by Escher and Simonett [8].
\end{abstract}

\section{Introduction}

Given a sufficiently smooth, compact hypersurface $\Omega_{0}=\boldsymbol{X}_{0}\left(M^{n}\right) \subset \mathbb{R}^{n+1}$, where $M^{n}$ is an $n$-dimensional manifold with (possibly empty) boundary, we are interested in finding a family of embeddings $\boldsymbol{X}: M^{n} \times[0, T) \rightarrow \mathbb{R}^{n+1}$ such that

$$
\frac{\partial \boldsymbol{X}}{\partial t}=(h-H) \boldsymbol{\nu}_{\Omega_{t}}, \quad \boldsymbol{X}(\cdot, 0)=\boldsymbol{X}_{0}, \quad h=\int_{M^{n}} H d \mu_{t}:=\frac{1}{\left|\Omega_{t}\right|} \int_{M^{n}} H d \mu_{t},
$$

where $H=\sum_{i=1}^{n} \kappa_{i}$ is the mean curvature and $\kappa_{i}$ are the principal curvatures of the hypersurface $\Omega_{t}=\boldsymbol{X}\left(M^{n}, t\right)=\boldsymbol{X}_{t}\left(M^{n}\right), \boldsymbol{\nu}_{\Omega_{t}}$ and $d \mu_{t}$ are a choice of unit normal and the induced measure of $\Omega_{t}$ respectively. Note that the flow (1.1) decreases surface area, $\left|\Omega_{t}\right|:=\int_{M^{n}} d \mu_{t}$, while preserving volume (see Section 2).

For compact, convex hypersurfaces without boundary it is known that a unique solution exists for all time and that the hypersurfaces converge to a sphere as $t \rightarrow \infty[10]$. Other results include average mean convex hypersurfaces with initially small traceless second fundamental form converging to spheres [11] and hypersurfaces that are graphs over spheres with a height function close to zero, in a certain function space, converging to spheres [8]. 
The case where the initial hypersurface has a boundary has also been studied. In this case it is assumed that $\Omega_{0}$ is smoothly embedded in the domain

$$
W=\left\{\boldsymbol{x} \in \mathbb{R}^{n+1}: 0<x_{n+1}<d\right\},
$$

with $d>0$ and $\partial \Omega_{0} \subset \partial W$. The volume enclosed by $\Omega_{0}$ and $W$ will be labelled $V$ and $\boldsymbol{\nu}_{\Omega_{t}}$ is chosen to point outward. The boundary conditions for the flow are then that $\Omega_{t}$ meets $\partial W$ orthogonally. Assuming $\Omega_{0}$ to be rotationally symmetric it was proved in [3] that the flow exists for all time and converges to a cylinder in $W$ of volume $V$ under the assumption

$$
\left|\Omega_{0}\right| \leq \frac{V}{d}
$$

This constraint ensures that the solution never touches the axis of rotation, so that no singularities develop. Short time existence of the flow can be proven using standard parabolic techniques for initially smooth hypersurfaces, however in this paper we are concerned with a larger class of initial hypersurfaces so need a more general existence theorem (see Section 2).

The main result of this paper is

Theorem 1.1. If $\Omega_{0}$ is a graph over the cylinder of radius $R$ and length $d, \mathscr{C}_{R, d}^{n}$, such that $R>\frac{d \sqrt{n-1}}{\pi}$ and with height function sufficiently small in $h_{\frac{\partial}{\partial z}}^{1+\beta}\left(\mathscr{C}_{R, d}^{n}\right)$ (see Section 2), for some $0<\beta<1$, then its flow by (1.1) exists for all time and converges exponentially fast to a cylinder as $t \rightarrow \infty$, with respect to the $C^{k}$ topology for any $k \in \mathbb{N}$.

An important point to note is that the limiting cylinder may not be the same as the cylinder $\mathscr{C}_{R, d}^{n}$, thus the cylinders are stable critical points of the PDE (1.1), but not asymptotically stable. An immediate consequence of this theorem is that there are non-axially symmetric hypersurfaces that converge to a cylinder under the flow (1.1), since there are non-axially symmetric hypersurfaces in any $h_{\frac{\partial}{\partial z}}^{1+\beta}$ neighborhood of a cylinder. Also note that the condition $R>\frac{d \sqrt{n-1}}{\pi}$ is of a similar nature to the condition (1.2); in fact for a cylinder (1.2) reduces to $R \geq n d$. In view of this, when considering the flow of axially-symmetric hypersurfaces that are close to a cylinder we reproduce the results of [3] under a weaker condition. The condition is also sharp, in the sense that if $R=\frac{d \sqrt{n-1}}{\pi}$ then there is a one-parameter family of unduloids, satisfying the boundary conditions, such that $\mathscr{C}_{R, d}^{n}$ is the only cylinder in the family, i.e., there are stationary solutions to the flow arbitrarily close to $\mathscr{C}_{R, d}^{n}$ that are not cylinders. 
In Section 2 of this paper, we convert the flow (1.1) to a PDE for the graph function and we show how this can be extended to a flow on the compact manifold $\mathscr{T}_{R}^{n}:=\mathscr{S}_{R}^{n-1} \times(-d, d]$, where we identify the ends at $-d$ and $d$. We will then introduce the function spaces and notation that will be used throughout the paper and present some important theorems about the flow, this includes a new existence result for non-smooth initial hypersurfaces. In Section 3 we consider the problem as an ODE on Banach spaces and determine the linearization of the speed as well as computing its spectrum. This then allows us to find a center manifold for the flow, which is proven to consist, locally, entirely of cylinders. Lastly exponential attractivity to this manifold is proven.

The author would like to thank Maria Athanassenas for her initial suggestion of working with cylinders, support, advice and help in preparing this paper, and Todd Oliynyk for his advice and encouragement in dealing with center manifolds. My thanks also to the referees and editor for their comments and suggestions. Finally to Monash University and the School of Mathematical Sciences for their support.

\section{Notation and preliminaries}

In this paper we consider $M^{n}=\mathscr{C}_{R, d}^{n}$, a given cylinder of radius $R$ and length $d$, and hypersurfaces that are normal graphs over $\mathscr{C}_{R, d}^{n}, \boldsymbol{X}_{\rho}(\boldsymbol{p})=$ $\boldsymbol{p}+\rho(\boldsymbol{p}) \boldsymbol{\nu}_{\mathscr{C}_{R, d}^{n}}(\boldsymbol{p}), \boldsymbol{p} \in \mathscr{C}_{R, d}^{n}$. We use the notation $\boldsymbol{q} \in \mathscr{S}_{R}^{n-1}$, the $(n-1)$ dimensional sphere of radius $R$, and $z \in[0, d]$, so that $(\boldsymbol{q}, z) \in \mathscr{C}_{R, d}^{n}$. The volume form on such a cylindrical graph will be denoted by $d \mu_{\rho}$ and we let $\mu(\rho)$ be the operator such that $d \mu_{\rho}=\mu(\rho) d z d \sigma$, where $d \sigma$ is the volume form on $\mathscr{S}_{R}^{n-1}$. We now give a representation for the mean curvature of a generic normal graph (see [7] for the spherical graph case).

Lemma 2.1. Let $g_{i j}$ and $h_{i j}$ be the metric and second fundamental form induced by the embedding $\boldsymbol{X}_{0}: M^{n} \rightarrow \mathbb{R}^{n+1}$ and consider the normal graph $\boldsymbol{X}_{\rho}=\boldsymbol{X}_{0}+\rho \boldsymbol{\nu}_{0}$, where $\rho: M^{n} \rightarrow \mathbb{R}$ is such that $\delta_{i}^{j}+\rho h_{i}^{j}$ is invertible. Define $\tilde{g}_{\rho}^{i j}$ to be the inverse of $\left(\delta_{i}^{k}+\rho h_{i}^{k}\right) g_{k l}\left(\delta_{j}^{l}+\rho h_{j}^{l}\right)$. The mean curvature of $\boldsymbol{X}_{\rho}\left(M^{n}\right)$ is given by the operator:

$$
\begin{aligned}
H(\rho)= & -L(\rho)^{-3}\left(L(\rho)^{2} \tilde{g}_{\rho}^{i j}-\tilde{g}_{\rho}^{i k} \tilde{g}_{\rho}^{j l} \frac{\partial \rho}{\partial x^{k}} \frac{\partial \rho}{\partial x^{l}}\right) \frac{\partial^{2} \rho}{\partial x^{i} \partial x^{j}}+L(\rho)^{-1} \tilde{g}_{\rho}^{i j}\left(\delta_{i}^{k}+\rho h_{i}^{k}\right) h_{k j} \\
& +L(\rho)^{-3}\left(\tilde{g}_{\rho}^{k l} \tilde{g}_{\rho}^{i j}\left(\delta_{l}^{p}+\rho h_{l}^{p}\right) h_{p j} \frac{\partial \rho}{\partial x^{i}}+\left(L(\rho)^{2} \tilde{g}_{\rho}^{i j}-\tilde{g}_{\rho}^{i q} \tilde{g}_{\rho}^{j l} \frac{\partial \rho}{\partial x^{q}} \frac{\partial \rho}{\partial x^{l}}\right) \Gamma_{i j}^{k}\right) \frac{\partial \rho}{\partial x^{k}} \\
& +L(\rho)^{-3}\left(L(\rho)^{2} \tilde{g}_{\rho}^{i j}-\tilde{g}_{\rho}^{i q} \tilde{g}_{\rho}^{j l} \frac{\partial \rho}{\partial x^{q}} \frac{\partial \rho}{\partial x^{l}}\right) \rho \tilde{g}_{\rho}^{k r}\left(\delta_{r}^{p}+\rho h_{r}^{p}\right) \nabla_{p} h_{i j} \frac{\partial \rho}{\partial x^{k}},
\end{aligned}
$$


where $L(\rho):=\sqrt{1+\tilde{g}_{\rho}^{i j} \frac{\partial \rho}{\partial x^{i}} \frac{\partial \rho}{\partial x^{j}}}, \Gamma_{i j}^{k}$ are the Christoffel symbols and $\nabla$ is the covariant derivative on $\boldsymbol{X}_{0}\left(M^{n}\right)$.

Proof. We can calculate the derivatives of the embedding by using the Gauss-Weingarten relations, $\frac{\partial \boldsymbol{\nu}}{\partial x^{i}}=h_{i}^{k} \frac{\partial \boldsymbol{X}}{\partial x^{k}}$ and $\frac{\partial^{2} \boldsymbol{X}}{\partial x^{i} \partial x^{j}}=\Gamma_{i j}^{k} \frac{\partial \boldsymbol{X}}{\partial x^{k}}-h_{i j} \boldsymbol{\nu}$ :

$$
\begin{aligned}
& \frac{\partial \boldsymbol{X}_{\rho}}{\partial x^{i}}=\left(\delta_{i}^{k}+\rho h_{i}^{k}\right) \frac{\partial \boldsymbol{X}_{0}}{\partial x^{k}}+\frac{\partial \rho}{\partial x^{i}} \boldsymbol{\nu}_{0}, \\
\frac{\partial^{2} \boldsymbol{X}_{\rho}}{\partial x^{i} \partial x^{j}}= & \left(h_{i}^{k} \frac{\partial \rho}{\partial x^{j}}+\rho \frac{\partial h_{i}^{k}}{\partial x^{j}}+\Gamma_{i j}^{k}+\rho h_{i}^{l} \Gamma_{l j}^{k}+h_{j}^{k} \frac{\partial \rho}{\partial x^{i}}\right) \frac{\partial \boldsymbol{X}_{0}}{\partial x^{k}} \\
& +\left(\frac{\partial^{2} \rho}{\partial x^{i} \partial x^{j}}-\left(\delta_{i}^{k}+\rho h_{i}^{k}\right) h_{k j}\right) \boldsymbol{\nu}_{0} \\
= & \left(h_{i}^{k} \frac{\partial \rho}{\partial x^{j}}+h_{j}^{k} \frac{\partial \rho}{\partial x^{i}}+\rho \nabla_{j} h_{i}^{k}+\left(\delta_{l}^{k}+\rho h_{l}^{k}\right) \Gamma_{i j}^{l}\right) \frac{\partial \boldsymbol{X}_{0}}{\partial x^{k}} \\
& +\left(\frac{\partial^{2} \rho}{\partial x^{i} \partial x^{j}}-\left(\delta_{i}^{k}+\rho h_{i}^{k}\right) h_{k j}\right) \boldsymbol{\nu}_{0} .
\end{aligned}
$$

The metric, $g_{\rho}$, of the hypersurface is therefore given by:

$$
g_{\rho i j}=\left(\delta_{i}^{k}+\rho h_{i}^{k}\right) g_{k l}\left(\delta_{j}^{l}+\rho h_{j}^{l}\right)+\frac{\partial \rho}{\partial x^{i}} \frac{\partial \rho}{\partial x^{j}},
$$

and using the definition of $\tilde{g}_{\rho}^{i j}$, the inverse metric of a normal graph can be written as:

$$
g_{\rho}^{i j}:=\left(g_{\rho}^{-1}\right)^{i j}=\tilde{g}_{\rho}^{i j}-L(\rho)^{-2} \tilde{g}_{\rho}^{i k} \tilde{g}_{\rho}^{j l} \frac{\partial \rho}{\partial x^{k}} \frac{\partial \rho}{\partial x^{l}} .
$$

The normal to the hypersurface is also easily verified to be:

$$
\boldsymbol{\nu}_{\rho}=L(\rho)^{-1}\left(\boldsymbol{\nu}_{0}-\tilde{g}_{\rho}^{r p}\left(\delta_{p}^{s}+\rho h_{p}^{s}\right) \frac{\partial \rho}{\partial x^{r}} \frac{\partial \boldsymbol{X}_{0}}{\partial x^{s}}\right)
$$

Calculating $-\boldsymbol{\nu}_{\rho} \cdot \frac{\partial^{2} \boldsymbol{X}_{\rho}}{\partial x^{i} \partial x^{j}}$ we obtain the second fundamental form, $A_{\rho}$, of $\boldsymbol{X}_{\rho}\left(M^{n}\right)$ :

$$
\begin{aligned}
h_{\rho i j}=L(\rho)^{-1}( & -\frac{\partial^{2} \rho}{\partial x^{i} \partial x^{j}}+\left(\delta_{i}^{k}+\rho h_{i}^{k}\right) h_{k j}+\tilde{g}_{\rho}^{r p}\left(\delta_{p}^{s}+\rho h_{p}^{s}\right) \frac{\partial \rho}{\partial x^{r}}\left(h_{i s} \frac{\partial \rho}{\partial x^{j}}+h_{j s} \frac{\partial \rho}{\partial x^{i}}\right) \\
& \left.+\Gamma_{i j}^{r} \frac{\partial \rho}{\partial x^{r}}+\rho \tilde{g}_{\rho}^{r p}\left(\delta_{p}^{s}+\rho h_{p}^{s}\right) \nabla_{j} h_{i s} \frac{\partial \rho}{\partial x^{r}}\right)
\end{aligned}
$$

so taking the trace with respect to $g_{\rho}$ and using $g_{\rho}^{i j} \frac{\partial \rho}{\partial x^{j}}=L(\rho)^{-2} \tilde{g}_{\rho}^{i j} \frac{\partial \rho}{\partial x^{j}}$ we get the result. 
Note that in the case $\boldsymbol{X}_{0}$ defines $\mathscr{C}_{R, d}^{n}$ the last line vanishes since $\nabla_{k} h_{i j}=$ 0 . We now proceed as in [8] and convert the flow to an evolution equation for the height function $\rho: \mathscr{C}_{R, d}^{n} \times[0, T) \rightarrow \mathbb{R}$. It is known that up to a tangential diffeomorphism the flow (1.1) is equivalent to

$$
\frac{\partial \rho}{\partial t}=L(\rho)\left(h_{\rho}-H(\rho)\right),\left.\quad \frac{\partial \rho}{\partial z}\right|_{z=0, d}=0, \quad \rho(\cdot, 0)=\rho_{0}
$$

where $h_{\rho}=f_{\mathscr{C}_{R, d}^{n}} H(\rho) d \mu_{\rho}$ (see [5]).

The presence of a boundary can make some details complicated, however the boundary conditions give us a natural extension to functions on $\mathscr{T}_{R}^{n}$. That is, for $\rho: \mathscr{C}_{R, d}^{n} \rightarrow \mathbb{R}$ that satisfies the boundary conditions we define $u_{\rho}: \mathscr{T}_{R}^{n} \rightarrow \mathbb{R}$ by:

$$
u_{\rho}(\boldsymbol{q}, z)=\left\{\begin{array}{cc}
\rho(\boldsymbol{q},-z) & z \in(-d, 0) \\
\rho(\boldsymbol{q}, z) & z \in[0, d]
\end{array}\right.
$$

We also extend $g_{i j}$ and $h_{i j}$ to $\mathscr{T}_{R}^{n}$ and the former becomes the metric on the manifold. We are then able to consider the flow

$$
\frac{\partial u}{\partial t}=L(u)\left(h_{u}-H(u)\right):=G(u), \quad u(\cdot, 0)=u_{0}
$$

where we now have $h_{u}=f_{\mathscr{T}_{R}^{n}} H(u) d \mu_{u}$ and we note that if $u$ has an even symmetry then $G(u)$ retains the symmetry. Therefore a solution to (2.2), with initial condition $u_{\rho_{0}}$, when restricted to $\mathscr{C}_{R, d}^{n} \subset \mathscr{T}_{R}^{n}$ will be a solution to $(2.1)$.

Throughout the paper we will be considering functions in the littleHölder spaces, which are defined for $\alpha \in(0,1), k \in \mathbb{N}$, an open set $U \subset \mathbb{R}^{n}$ and a multi-index $\beta=\left(\beta_{1}, \ldots, \beta_{n}\right)$ with $|\beta|=\sum_{i=1}^{n} \beta_{i}$ as follows (see [12]):

$$
\begin{aligned}
h^{\alpha}(\bar{U}) & =\left\{\rho \in C^{\alpha}(\bar{U}): \lim _{r \rightarrow 0} \sup _{x, y \in \bar{U}, 0<|x-y|<r} \frac{|\rho(x)-\rho(y)|}{|x-y|^{\alpha}}=0\right\}, \\
h^{k+\alpha}(\bar{U}) & =\left\{\rho \in C^{k+\alpha}(\bar{U}): D^{\beta} \rho \in h^{\alpha}(\bar{U}) \text { for all } \beta,|\beta|=k\right\},
\end{aligned}
$$


where $D$ is the derivative operator on $\mathbb{R}^{n}$ and $C^{\alpha}, C^{k+\alpha}$ are the Hölder spaces:

$$
\begin{aligned}
C^{\alpha}(\bar{U}) & =\left\{\rho \in C(\bar{U}): \sup _{x, y \in \bar{U}, x \neq y} \frac{|\rho(x)-\rho(y)|}{|x-y|^{\alpha}}<\infty\right\}, \\
C^{k+\alpha}(\bar{U}) & =\left\{\rho \in C^{k}(\bar{U}): D^{\beta} \rho \in C^{\alpha}(\bar{U}) \text { for all } \beta,|\beta|=k\right\} .
\end{aligned}
$$

The norms on the little-Hölder spaces are the same as those on the Hölder spaces,

$$
\|\rho\|_{h^{k+\alpha}(\bar{U})}=\|\rho\|_{C^{k+\alpha}(\bar{U})}=\|\rho\|_{C^{k}(\bar{U})}+\sum_{|\beta|=k} \sup _{x, y \in \bar{U}, x \neq y} \frac{\left|D^{\beta} \rho(x)-D^{\beta} \rho(y)\right|}{|x-y|^{\alpha}},
$$

where

$$
\|\rho\|_{C^{k}(\bar{U})}=\sum_{|\beta| \leq k} \sup _{x \in \bar{U}}\left|D^{\beta} \rho(x)\right| .
$$

The little-Hölder spaces can be extended to a manifold by means of the atlas of $\mathscr{C}_{R, d}^{n}$ and $\mathscr{T}_{R}^{n}$. In addition, it is known that the little-Hölder spaces are the continuous interpolation spaces between themselves (see [9] Equation 19), that is for real numbers $0<\alpha<\beta$

$$
\left(h^{\alpha}\left(\mathscr{T}_{R}^{n}\right), h^{\beta}\left(\mathscr{T}_{R}^{n}\right)\right)_{\theta}=h^{(\beta-\alpha) \theta+\alpha}\left(\mathscr{T}_{R}^{n}\right),
$$

where $(\beta-\alpha) \theta+\alpha \notin \mathbb{N}$ and $(\cdot, \cdot)$ is an interpolation functor for each $\theta \in$ $(0,1)$ and is defined for $Y \subset X$ as follows:

$$
\begin{aligned}
(X, Y)_{\theta} & =\left\{x \in X: \lim _{t \rightarrow 0} t^{-\theta} K(t, x, X, Y)=0\right\}, \\
K(t, x, X, Y) & =\inf _{a \in Y}\left(\|x-a\|_{X}+t\|a\|_{Y}\right) .
\end{aligned}
$$

To simplify the notation involving the boundary conditions, let $Y\left(\mathscr{C}_{R, d}^{n}\right)$ represent any function space on the cylinder and set

$$
Y_{\frac{\partial}{\partial z}}\left(\mathscr{C}_{R, d}^{n}\right)=\left\{\rho \in Y\left(\mathscr{C}_{R, d}^{n}\right):\left.\frac{\partial \rho}{\partial z}\right|_{z=0, d}=0\right\}
$$

For an operator between function spaces $G: Y \rightarrow \tilde{Y}$ we denote the Fréchet derivative by $\partial G$. A linear operator, $A: Y \subset X \rightarrow X$, is called sectorial if there exist $\theta \in\left(\frac{\pi}{2}, \pi\right)$ and $\omega \in \mathbb{R}$ and $M>0$ such that 
Motion by volume preserving mean curvature flow near cylinders 879

(i) $\rho(A) \supset S_{\theta, \omega}=\{\lambda \in \mathbb{C}: \lambda \neq \omega,|\arg (\lambda-\omega)|<\theta\}$,

(ii) $\|R(\lambda, A)\|_{L(X)} \leq \frac{M}{|\lambda-\omega|}$ for all $\lambda \in S_{\theta, \omega}$,

here $\rho(A)$ is the resolvent set, $R(\lambda, A)=(\lambda I-A)^{-1}$ is the resolvent operator and $\|\cdot\|_{L(X)}$ is the standard linear operator norm (see [12]).

The following are some facts about the flow.

Lemma 2.2. For an initially smooth, compact hypersurface with boundary contained in $\partial W$, the flow (1.1) together with Neumann boundary condition:

(i) preserves the enclosed volume $V$,

(ii) decreases the surface area $\left|\Omega_{t}\right|$.

Proof. Both parts are covered in [3]. Using the divergence theorem and setting $E_{t} \subset W$ to be the $(n+1)$-dimensional space with boundary $\Omega_{t}$ we have the first part of the lemma as follows

$$
\frac{d V}{d t}=\int_{E_{t}} \operatorname{div} \frac{\partial \boldsymbol{X}}{\partial t} d \boldsymbol{x}=\int_{\partial E_{t}} \frac{\partial \boldsymbol{X}}{\partial t} \cdot \boldsymbol{\nu}_{\partial E_{t}} d S=\int_{M^{n}}(h-H) d \mu_{t}=0,
$$

where the integral over the part of the boundary contained in $\partial W$ vanishes due to the boundary conditions. The second statement is through a direct calculation of the evolution equation for the area

$$
\frac{d\left|\Omega_{t}\right|}{d t}=-\int_{M^{n}}(H-h)^{2} d \mu_{t}
$$

Note that Lemma 2.2 is also true for hypersurfaces without boundary. We now are able to prove an existence theorem for hypersurfaces defined by a graph function in $h_{\frac{\partial}{\partial z}}^{1+\beta}\left(\mathscr{C}_{R, d}^{n}\right)$.

Theorem 2.3. Given a graph over $\mathscr{C}_{R, d}^{n}, \Omega_{0}$, with height function $\rho_{0} \in h_{\frac{\partial}{\partial z}}^{1+\beta}$ $\left(\mathscr{C}_{R, d}^{n}\right)$, some $\beta \in(0,1)$, the flow (1.1) together with the Neumann boundary conditions has a unique classical solution $\left\{\Omega_{t}: t \in[0, T)\right\}$ for some $T>0$. Each hypersurface $\Omega_{t}$ is of class $C^{\infty}$ for $t \in(0, T)$. Moreover, the mapping $t \rightarrow \Omega_{t}$ is continuous on $[0, T)$ with respect to the $h^{1+\beta}$-topology and smooth on $(0, T)$ with respect to the $C^{\infty}$-topology. 
Proof. We first fix $0<\alpha<\beta_{0}<\beta$ and consider (2.2) with initial condition $u_{\rho_{0}} \in h^{1+\beta}\left(\mathscr{T}_{R}^{n}\right)$ as an ordinary differential equation between Banach spaces:

$$
u^{\prime}(t)=G(u(t)), \quad 0<t<T, \quad u(0)=u_{\rho_{0}} \in h^{1+\beta}\left(\mathscr{T}_{R}^{n}\right),
$$

where $G: h^{2+\alpha}\left(\mathscr{T}_{R}^{n}\right) \rightarrow h^{\alpha}\left(\mathscr{T}_{R}^{n}\right)$. We follow the proof for the case of compact, closed, connected, embedded hypersurfaces by Escher and Simonett in [8]. Set $U:=\left\{u \in h^{1+\beta_{0}}\left(\mathscr{T}_{R}^{n}\right): u>-R\right\}$, then by Lemma 2.1 there is the decomposition $H(u)=P(u) u+Q(u)$, for $u \in U \cap h^{2+\alpha}\left(\mathscr{T}_{R}^{n}\right)$, with

$$
P(u):=-L(u)^{-3}\left(L(u)^{2} \tilde{g}_{u}^{i j}-\tilde{g}_{u}^{i k} \tilde{g}_{u}^{j l} \frac{\partial u}{\partial x^{k}} \frac{\partial u}{\partial x^{l}}\right) \frac{\partial^{2}}{\partial x^{i} \partial x^{j}},
$$

a uniformly elliptic operator and

$$
\begin{aligned}
Q(u):= & L(u)^{-3}\left(\tilde{g}_{u}^{k l} \tilde{g}_{u}^{i j}\left(\delta_{l}^{p}+u h_{l}^{p}\right) h_{p j} \frac{\partial u}{\partial x^{i}}+\left(L(u)^{2} \tilde{g}_{u}^{i j}-\tilde{g}_{u}^{i q} \tilde{g}_{u}^{j l} \frac{\partial u}{\partial x^{q}} \frac{\partial u}{\partial x^{l}}\right) \Gamma_{i j}^{k}\right) \frac{\partial u}{\partial x^{k}} \\
& +L(u)^{-1} \tilde{g}_{u}^{i j}\left(\delta_{i}^{k}+u h_{i}^{k}\right) h_{k j} .
\end{aligned}
$$

These maps are also smooth, i.e., $P \in C^{\infty}\left(U, \mathcal{L}\left(h^{2+\alpha}\left(\mathscr{T}_{R}^{n}\right), h^{\alpha}\left(\mathscr{T}_{R}^{n}\right)\right)\right)$ and $Q \in C^{\infty}\left(U, h^{\beta_{0}}\left(\mathscr{T}_{R}^{n}\right)\right)$. If we define

$$
\bar{G}_{u} v=L(u)\left(P(u) v-f_{\mathscr{T}_{R}^{n}} P(u) v d \mu_{u}\right),
$$

and

$$
f(u)=G(u)+\bar{G}_{u} u=L(u)\left(f_{\mathscr{T}_{R}^{n}} Q(u) d \mu_{u}-Q(u)\right),
$$

we have that $f \in C^{\infty}\left(U, h^{\beta_{0}}\left(\mathscr{T}_{R}^{n}\right)\right)$. Following Lemma 3.4 in [9] we use that since $L(u) P(u)$ is uniformly elliptic for all $u \in U$ there exist $\omega, C>0$ such that $\lambda I+L(u) P(u): h^{2+\alpha}\left(\mathscr{T}_{R}^{n}\right) \rightarrow h^{\alpha}\left(\mathscr{T}_{R}^{n}\right)$ is a linear isomorphism and satisfies the Schauder estimate:

$$
\|v\|_{h^{2+l+\alpha}} \leq C\|(\lambda I+L(u) P(u)) v\|_{h^{l+\alpha}} \text {, for all } v \in h^{2+l+\alpha}\left(\mathscr{T}_{R}^{n}\right),
$$

for $\operatorname{Re}(\lambda)>\omega$ and any integer $l \geq 0$, see also Theorems 27 and 37 in [4]. Therefore by Remark 1.2.1(a) and Theorem 1.2.2 in [2] there exists $B_{l}>0$ such that

$$
|\lambda|\|v\|_{h^{l+\alpha}}+\|v\|_{h^{2+l+\alpha}} \leq B_{l}\|(\lambda I+L(u) P(u)) v\|_{h^{l+\alpha}}, \text { for all } v \in h^{2+l+\alpha}\left(\mathscr{T}_{R}^{n}\right),
$$

for $\operatorname{Re}(\lambda)>\omega$ and hence $-L(u) P(u)$ generates an analytic semigroup in $h^{l+\alpha}\left(\mathscr{T}_{R}^{n}\right)$. The same perturbation argument as in [8] can then be used, 
Motion by volume preserving mean curvature flow near cylinders 881

since it is clear that

$$
\left\|L(u) f_{\mathscr{T}_{R}^{n}} P(u) v d \mu_{u}\right\|_{h^{\alpha}}=\left|f_{\mathscr{T}_{R}^{n}} P(u) v d \mu_{u}\right|\|L(u)\|_{h^{\alpha}} \leq C(u)\|v\|_{C^{2}},
$$

to conclude that $-\bar{G}_{u}$ generates an analytic semigroup in $h^{l+\alpha}\left(\mathscr{T}_{R}^{n}\right)$. Applying Theorem 12.1 in [1] we obtain a unique solution to $(2.4), u(t)$, such that

$$
\begin{aligned}
u \in & C\left([0, \delta), U \cap h^{1+\beta}\left(\mathscr{T}_{R}^{n}\right)\right) \cap C^{(1+\beta-\alpha) / 2}\left([0, \delta), h^{\alpha}\left(\mathscr{T}_{R}^{n}\right)\right) \\
& \cap C\left((0, \delta), h^{2+\alpha}\left(\mathscr{T}_{R}^{n}\right)\right) .
\end{aligned}
$$

Since $-\bar{G}_{u}$ generates an analytic semigroup in $h^{l+\alpha}\left(\mathscr{T}_{R}^{n}\right)$ for any $l \geq 0$, the same bootstrapping arguments as in the proof of Theorem 1 in [6] give us the smoothness of $u$. By setting $\rho(t)=\left.u(t)\right|_{\mathscr{C}_{R, d}^{n}}$ we obtain the result.

\section{Stability around cylinders}

In order to determine the stability of the cylinder we need to investigate the linearization of $G$ about zero.

Lemma 3.1. For the linearization, $\partial G(0)$, of $G$ it holds

$$
\partial G(0) v=\left(\frac{n-1}{R^{2}}+\Delta_{\mathscr{T}_{R}^{n}}\right) v-\frac{n-1}{R^{2}} f_{\mathscr{T}_{R}^{n}} v d z d \sigma
$$

for $v \in h^{2+\alpha}\left(\mathscr{T}_{R}^{n}\right)$.

Proof. Firstly note that $L(0)=1$ and that $\partial L(0)=0$. Hence for $v \in$ $h^{2+\alpha}\left(\mathscr{T}_{R}^{n}\right)$

$$
\begin{aligned}
\left.\partial h_{u}\right|_{u=0} v & =\left.\partial\left(\frac{1}{\int_{\mathscr{T}_{R}^{n}} \mu(u) d z d \sigma} \int_{\mathscr{T}_{R}} H(u) \mu(u) d z d \sigma\right)\right|_{u=0} v \\
& =\frac{1}{\left(\int_{\mathscr{T}_{R}^{n}} d z d \sigma\right)^{2}}\left(\left.\int_{\mathscr{T}_{R}^{n}} d z d \sigma \partial\left(\int_{\mathscr{T}_{R}^{n}} H(u) \mu(u) d z d \sigma\right)\right|_{u=0} v\right. \\
& \left.\quad-\left.\int_{\mathscr{T}_{R}^{n}} H(0) d z d \sigma \partial\left(\int_{\mathscr{T}_{R}^{n}} \mu(u) d z d \sigma\right)\right|_{u=0} v\right) \\
& =\frac{1}{\left|\mathscr{T}_{R}^{n}\right|}\left(\int_{\mathscr{T}_{R}^{n}} \partial H(0) v+H(0) \partial \mu(0) v d z d \sigma-H(0) \int_{\mathscr{T}_{R}^{n}} \partial \mu(0) v d z d \sigma\right) \\
& =f_{\mathscr{T}_{R}^{n}} \partial H(0) v d z d \sigma .
\end{aligned}
$$


We can use Lemma 2.1 to calculate the linearization of $H(u)$

$$
\partial H(0)=-g^{i j} \frac{\partial^{2}}{\partial x^{i} \partial x^{j}}+g^{i j} \Gamma_{i j}^{k} \frac{\partial}{\partial x^{k}}-h^{i j} h_{i j}
$$

where we have used the above results for $L(u)$ and the formula

$$
\partial \tilde{g}_{u}^{i j}=-2 \tilde{g}_{u}^{i k}\left(\delta_{k}^{q}+u h_{k}^{q}\right) h_{q l} \tilde{g}_{u}^{l j} .
$$

Combining these results gives, for $v \in h^{2+\alpha}\left(\mathscr{T}_{R}^{n}\right)$,

$$
\partial G(0) u=\left(\frac{n-1}{R^{2}}+\Delta_{\mathscr{T}_{R}^{n}}\right) u-f_{\mathscr{T}_{R}^{n}}\left(\frac{n-1}{R^{2}}+\Delta_{\mathscr{T}_{R}^{n}}\right) u d z d \sigma .
$$

The divergence theorem gives the result.

As we are considering the flow locally about $u=0$ it is convenient to rewrite (2.4) highlighting the dominant linear part

$$
u^{\prime}(t)=\partial G(0) u(t)+\tilde{G}(u(t)), \quad \tilde{G}(u):=G(u)-\partial G(0) u .
$$

Lemma 3.2. If $R>\frac{d \sqrt{n-1}}{\pi}$ then the spectrum $\sigma(\partial G(0))$ of $\partial G(0)$ consists of a sequence of isolated non-positive eigenvalues where the multiplicity of the 0 eigenvalue is $n+1$.

Proof. First note that $\partial G(0): h^{2+\alpha}\left(\mathscr{T}_{R}^{n}\right) \rightarrow h^{\alpha}\left(\mathscr{T}_{R}^{n}\right)$ and since $h^{2+\alpha}\left(\mathscr{T}_{R}^{n}\right)$ is compactly embedded in $h^{\alpha}\left(\mathscr{T}_{R}^{n}\right)$ the spectrum consists solely of eigenvalues. To characterize the spectrum of $\partial G(0)$ we first look at the spectrum of

$$
\tilde{A}:=\frac{n-1}{R^{2}}+\Delta_{\mathscr{T}_{R}^{n}}
$$

In the following we deal with the natural complexification without distinguishing it notationally. The operator $\tilde{A}$ is self adjoint with respect to the $L_{2}$ inner product on $h^{2+\alpha}\left(\mathscr{T}_{R}^{n}\right)$, this means that the spectrum consists of real numbers. For an eigenfunction $v$ of $\tilde{A}$ assume that $v(\boldsymbol{q}, z)=X(\boldsymbol{q}) Z(z)$, so using $\Delta_{\mathscr{T}_{R}^{n}}=\Delta_{\mathscr{S}_{R}^{n-1}}+\frac{\partial^{2}}{\partial z^{2}}$,

$$
\left(\frac{n-1}{R^{2}}+\Delta_{\mathscr{S}_{R}^{n-1}}+\frac{\partial^{2}}{\partial z^{2}}\right) X(\boldsymbol{q}) Z(z)=\lambda X(\boldsymbol{q}) Z(z) .
$$

So, after expanding,

$$
Z(z) \Delta_{\mathscr{S}_{R}^{n-1}} X(\boldsymbol{q})+X(\boldsymbol{q}) Z^{\prime \prime}(z)+\left(\frac{n-1}{R^{2}}-\lambda\right) X(\boldsymbol{q}) Z(z)=0 .
$$


Motion by volume preserving mean curvature flow near cylinders 883

Therefore

$$
-\frac{\Delta_{\mathscr{S}_{R}^{n-1}} X(\boldsymbol{q})}{X(\boldsymbol{q})}=\frac{Z^{\prime \prime}(z)}{Z(z)}+\left(\frac{n-1}{R^{2}}-\lambda\right) .
$$

Setting both sides equal to a constant $\xi \in \mathbb{R}$

$$
\Delta_{\mathscr{S}_{R}^{n-1}} X(\boldsymbol{q})=-\xi X(\boldsymbol{q})
$$

The eigenvalues of the Laplacian on $\mathscr{S}_{R}^{n-1}$ are well known to be $\frac{-l(l+n-2)}{R^{2}}$ for $l \in \mathbb{N} \cup\{0\}$, with eigenfunctions the spherical harmonics of order $l, X_{l, p}(\boldsymbol{q})=$ $Y_{l, p}(\boldsymbol{q}), 1 \leq p \leq M_{l}$, where

$$
M_{l}=\left\{\begin{array}{cc}
\left(\begin{array}{c}
l+n-1 \\
n-1
\end{array}\right)-\left(\begin{array}{c}
l+n-3 \\
n-1
\end{array}\right) & l \geq 2 \\
\left(\begin{array}{c}
l+n-1 \\
n-1
\end{array}\right) & l \in\{0,1\}
\end{array} .\right.
$$

Therefore, as $\xi_{l}=\frac{l(l+n-2)}{R^{2}}$, the other separation equation becomes

$$
Z^{\prime \prime}(z)=\left(\lambda+\frac{l(l+n-2)-(n-1)}{R^{2}}\right) Z(z),
$$

with $\lim _{z \rightarrow-d} Z(z)=Z(d)$ and $\lim _{z \rightarrow-d} Z^{\prime}(z)=Z^{\prime}(d)$ since $v \in h^{2+\alpha}\left(\mathscr{T}_{R}^{n}\right)$. The eigenvalues and eigenfunctions to the second separation equation are given by

$$
\lambda_{l, m}=-\left(\frac{m^{2} \pi^{2}}{d^{2}}+\frac{(l-1)(l+n-1)}{R^{2}}\right), \quad m, l \in \mathbb{N} \cup\{0\}
$$

and

$$
Z_{m, 1}(z)=\cos \left(\frac{m \pi z}{d}\right), \quad Z_{m, 2}(z)=\sin \left(\frac{m \pi z}{d}\right) .
$$

So $\lambda_{l, m}$ are the eigenvalues of $\tilde{A}$ and eigenfunctions spanned by

$$
v_{l, p, m, 1}(\boldsymbol{q}, z)=\cos \left(\frac{m \pi z}{d}\right) Y_{l, p}(\boldsymbol{q}), \quad v_{l, p, m, 2}(\boldsymbol{q}, z)=\sin \left(\frac{m \pi z}{d}\right) Y_{l, p}(\boldsymbol{q}),
$$

for $1 \leq p \leq M_{l}$. Since $v_{l, p, 0,2}=0$ we drop the final index in the case of $m=0$ and set $v_{l, p, 0,1}(\boldsymbol{q}, z)=v_{l, p, 0}(\boldsymbol{q}, z)=Y_{l, p}(\boldsymbol{q})$. Also, since the spherical harmonics are dense in the continuous functions on $\mathscr{S}_{R}^{n-1}$ and the trigonometric functions are dense in the continuous functions on $(-d, d]$ with associated ends (i.e., functions with period $2 d$ ), we have found every eigenfunction of $\tilde{A}$. 
Returning to the spectrum of $\partial G(0), v_{0,1,0}=1$ is still an eigenfunction but with eigenvalue $\lambda_{0,0}=0$. The operator $\partial G(0)$ is also self adjoint with respect to the $L_{2}$ inner product on $h^{2+\alpha}\left(\mathscr{T}_{R}^{n}\right)$. Therefore we need only consider other eigenfunctions, orthogonal to $v_{0,1,0}=1$, in order to characterize the spectrum. This means that for an eigenfunction $v$

$$
\int_{\mathscr{T}_{R}^{n}} v d z d \sigma=0,
$$

hence by Lemma 3.1, $\partial G(0) v=\tilde{A} v$. The remaining eigenfunctions of $\partial G(0)$ are then the remaining eigenfunctions of $\tilde{A}$, with the same eigenvalues. Therefore for $m, l \in \mathbb{N} \cup\{0\}$ and $m^{2}+l^{2} \geq 1$ the eigenvalues are

$$
\lambda_{l, m}=-\left(\frac{m^{2} \pi^{2}}{d^{2}}+\frac{(l-1)(l+n-1)}{R^{2}}\right),
$$

with eigenfunctions

$$
v_{l, p, m, 1}(\boldsymbol{q}, z)=\cos \left(\frac{m \pi z}{d}\right) Y_{l, p}(\boldsymbol{q}), \quad v_{l, p, m, 2}(\boldsymbol{q}, z)=\sin \left(\frac{m \pi z}{d}\right) Y_{l, p}(\boldsymbol{q}),
$$

for $1 \leq p \leq M_{l}$. It is clear from these expressions that $\lambda_{1,0}=0$, and we relabel the constant eigenfunction as $v_{1,0,0}=1$ for convenience, so zero is an eigenvalue of multiplicity at least $M_{1}+1=n+1$. If $m \in \mathbb{N}$ we have $\lambda_{1, m}<0$. Also if $l \geq 2$ and $m \in \mathbb{N} \cup 0$ then $\lambda_{l, m}<0$. The last case is for $l=0$ and $m \geq 1$ where the eigenvalues are bounded above by $\lambda_{0,1}$ :

$$
\lambda_{0, m}=-\left(\frac{m^{2} \pi^{2}}{d^{2}}-\frac{n-1}{R^{2}}\right) \leq-\left(\frac{\pi^{2}}{d^{2}}-\frac{n-1}{R^{2}}\right)=\lambda_{0,1} .
$$

These eigenvalues are all negative under the condition $R>\frac{d \sqrt{n-1}}{\pi}$ given in the statement of the theorem.

In what follows, we set $P_{0}$ to be the projection from $h^{\alpha}\left(\mathscr{T}_{R}^{n}\right)$ onto the $\lambda=0$ eigenspace given by

$$
P_{0} u:=\sum_{p=0}^{n}\left\langle u, v_{1, p, 0}\right\rangle v_{1, p, 0},
$$

where we use $\langle\cdot, \cdot\rangle$ to denote the $L_{2}$ inner product on $h^{\alpha}\left(\mathscr{T}_{R}^{n}\right)$. Because $\partial G(0)$ is self adjoint with respect to this inner product, $P_{0} \partial G(0) u=$ $\partial G(0) P_{0} u=0$ for every $u \in h^{2+\alpha}\left(\mathscr{T}_{R}^{n}\right)$. Due to this $h^{2+\alpha}\left(\mathscr{T}_{R}^{n}\right)$ can be split 
into the subspaces $X^{c}=P_{0}\left(h^{2+\alpha}\left(\mathscr{T}_{R}^{n}\right)\right)$ and $X^{s}=\left(I-P_{0}\right)\left(h^{2+\alpha}\left(\mathscr{T}_{R}^{n}\right)\right)$, called the center subspace and stable subspace respectively. We are now in a position to apply Theorem 9.2.2 in [12].

Theorem 3.3. For any $k \in \mathbb{N}$, there is a function $\gamma \in C^{k-1}\left(X^{c}, X^{s}\right)$, such that $\gamma^{(k-1)}$ is Lipschitz continuous, $\gamma(0)=\partial \gamma(0)=0$ and $\mathcal{M}^{c}=\operatorname{graph}(\gamma)$ is a locally invariant manifold of Equation (3.2) with dimension $n+1$, provided $R>\frac{d \sqrt{n-1}}{\pi}$.

Note that by locally invariant it is meant that there exists a neighborhood of zero, $\Lambda \subset X^{c}$, such that if $u_{0} \in \operatorname{graph}\left(\left.\gamma\right|_{\Lambda}\right)$ then the solution to (3.2) is in $\operatorname{graph}\left(\left.\gamma\right|_{\Lambda}\right)$ for all time or until $P_{0} u(t) \notin \Lambda$.

Proof. In order to know that the assumptions of Theorem 9.2.2 in [12] are satisfied in our case, we use Equation (2.3) to show that for any $\alpha_{0}$ satisfying $0<\alpha_{0}<\alpha$ the following holds

$$
\left(h^{\alpha_{0}}\left(\mathscr{T}_{R}^{n}\right), h^{2+\alpha_{0}}\left(\mathscr{T}_{R}^{n}\right)\right)_{\frac{\alpha-\alpha_{0}}{2}}=h^{\alpha}\left(\mathscr{T}_{R}^{n}\right) .
$$

The assumptions are therefore satisfied by proving there exists a neighborhood of zero, $O \subset h^{2+\alpha}\left(\mathscr{T}_{R}^{n}\right)$, such that $\tilde{G} \in C^{\infty}\left(O, h^{\alpha}\left(\mathscr{T}_{R}^{n}\right)\right)$ and $\partial G(0)$ is the part in $h^{\alpha}\left(\mathscr{T}_{R}^{n}\right)$ of a sectorial operator $A: h^{2+\alpha_{0}}\left(\mathscr{T}_{R}^{n}\right) \rightarrow h^{\alpha_{0}}\left(\mathscr{T}_{R}^{n}\right)$. By Lemma 2.1 we have that $\tilde{G}$ is a smooth operator inside $U$.

We also have that $\partial H(0)=-\left(\frac{n-1}{R^{2}}+\Delta_{\mathscr{T}_{R}^{n}}\right)$ is uniformly elliptic, so by the same arguments as in the proof of Theorem 2.3 we obtain $\omega, M>0$ such that

$$
|\lambda|\|v\|_{h^{\alpha_{0}}}+\|v\|_{h^{2+\alpha_{0}}} \leq M\|(\lambda I+\partial H(0)) v\|_{h^{\alpha_{0}}}, \text { for all } v \in h^{2+\alpha_{0}}\left(\mathscr{T}_{R}^{n}\right),
$$

for $\operatorname{Re}(\lambda)>\omega$, hence its negative is sectorial as a mapping from $h^{2+\alpha_{0}}\left(\mathscr{T}_{R}^{n}\right)$ into $h^{\alpha_{0}}\left(\mathscr{T}_{R}^{n}\right)$, by Proposition 2.1.11 in [12]. Now the operator

$$
\begin{aligned}
& A: h^{2+\alpha_{0}}\left(\mathscr{T}_{R}^{n}\right) \rightarrow h^{\alpha_{0}}\left(\mathscr{T}_{R}^{n}\right), \\
& A v=\left(\frac{n-1}{R^{2}}+\Delta_{\mathscr{T}_{R}^{n}}\right) v-\frac{n-1}{R^{2}} f_{\mathscr{T}_{R}^{n}} v d z d \sigma,
\end{aligned}
$$

can easily be seen to be sectorial, as it is a perturbation of $-\partial H(0)$ by a function bounded in $h^{\alpha_{0}}\left(\mathscr{T}_{R}^{n}\right)$, see Proposition 2.4.1 (i) in [12]. Therefore the assumptions are satisfied with $O=U$. 
We now set

$$
\mathcal{S}:=\left\{u \in h^{2+\alpha}\left(\mathscr{T}_{R}^{n}\right): u=u_{\rho} \text { and } \operatorname{graph}(\rho) \text { is a cylinder }\right\}
$$

and note that if $u \in \mathcal{S}$ then it is an equilibria of (3.2).

Lemma 3.4. $\mathcal{M}^{c}$ coincides with the set $\mathcal{S}$ on a neighborhood of zero, provided $R>\frac{d \sqrt{n-1}}{\pi}$.

Proof. Firstly, note that due to Theorem 2.4 in [13], Theorem 2.3 in [14] can be applied to conclude $\mathcal{M}^{c}$ contains all equilibria of (3.2) with $P_{0} u \in \Lambda$. Also note, the center manifold, $\mathcal{M}^{c}$, is defined differently in [14] as compared to [12], however they can be seen to be equal on $\Lambda$. The rest of the proof follows from [8], where $u \in \mathcal{S}$ is formulated in terms of eigenfunctions:

$$
u(\boldsymbol{y})=\sum_{p=1}^{n} y_{p} v_{1, p, 0}-R+\sqrt{\left(\sum_{p=1}^{n} y_{p} v_{1, p, 0}\right)^{2}+\left(R+y_{0}\right)^{2}-\sum_{p=1}^{n} y_{p}^{2}}
$$

where, if we consider the hypersurface $\operatorname{graph}(\rho) \subset \mathbb{R}^{n+1}$, where $u=u_{\rho}$, $\left(y_{1}, \ldots, y_{n}, 0\right) \in \mathbb{R}^{n+1}$ is the point of intersection of the axis of rotation with the $x_{n+1}$-plane and $y_{0}:=R^{\prime}-R$ the difference between its radius and the radius of $\mathscr{T}_{R}^{n}$. This map is smooth on a neighborhood, $\mathrm{O}_{2}$, of $0 \in \mathbb{R}^{n+1}$ and its derivative at zero is given by

$$
\partial u(0) \boldsymbol{y}=\sum_{p=0}^{n} y_{p} v_{1, p, 0}, \quad \boldsymbol{y} \in \mathbb{R}^{n+1} .
$$

The map taking $\boldsymbol{y}$ to the coordinates of $P_{0} u(\boldsymbol{y})$ with respect to the basis $v_{1, p, 0}$, is then found to have derivative at zero equal to the identity and hence is a diffeomorphism from $O_{2}$ onto its image, $\Lambda_{1}$, possibly making $O_{2}$ smaller. This means that $\mathcal{S}$ is locally a graph over $X^{c}$ with graph function $\left(I-P_{0}\right) u \circ\left(P_{0} u\right)^{-1}: \Lambda_{1} \rightarrow X^{s}$. Since we already have that $\left.\mathcal{S}\right|_{\Lambda} \subset \mathcal{M}^{c}, \mathcal{S}$ and $\mathcal{M}^{c}$ coincide on $\Lambda_{2}:=\Lambda_{1} \cap \Lambda$.

We now prove the main result.

Proof of Theorem 1.1. We again will prove the result for Equation (3.2) and hence obtain it for Equation (2.1). As in [8] we can use the same arguments in [7] to prove the following result. Given $k \in \mathbb{N}$ and $\omega \in\left(0,-\max \left(\lambda_{0,1}, \lambda_{2,0}\right)\right)$, there exists a neighborhood $O_{3}(k, \omega)$ of $0 \in h^{1+\beta}\left(\mathscr{T}_{R}^{n}\right)$ with the property: 
Given $u_{0} \in O_{3}$, the solution $u(t)$ of (2.4) exists globally and there exist $c(k, \omega)>0, T(k, \omega)>0$, and a unique $z_{0} \in \Lambda_{2}$ such that

$$
\left\|u(t)-\left(z_{0}+\gamma\left(z_{0}\right)\right)\right\|_{C^{k}} \leq c e^{-\omega t}\left\|\left(I-P_{0}\right) u_{0}-\gamma\left(P_{0} u_{0}\right)\right\|_{h^{1+\beta}},
$$

for $t>T$; here $z_{0}$ and $\gamma\left(z_{0}\right)$ are added in $C^{k}\left(\mathscr{T}_{R}^{n}\right)$. This proves that $u(t)$ converges exponentially fast to an element $u_{\infty}:=z_{0}+\left.\gamma\left(z_{0}\right) \in \mathcal{M}^{c}\right|_{\Lambda_{2}}$. Since $\left\|u_{\rho_{0}}\right\|_{h^{1+\beta}}$ is controlled by $\left\|\rho_{0}\right\|_{h^{1+\beta}}$ for any $\rho_{0} \in h_{\frac{\partial}{\partial z}}^{1+\beta}\left(\mathscr{C}_{R, d}^{n}\right)$, there exists a neighborhood of zero in $h_{\frac{\partial}{\partial z}}^{1+\beta}\left(\mathscr{C}_{R, d}^{n}\right)$ such that if $\rho_{0}$ is in this neighborhood then $u_{\rho_{0}} \in O_{3}$. Therefore $\rho(t)=\left.u(t)\right|_{\mathscr{C}_{R, d}^{n}}$ converges exponentially fast to $u_{\infty} \mid \mathscr{C}_{R, d}^{n}$, whose graph is a cylinder.

Corollary 3.5. Let $\Omega_{0}$ be a graph over a cylinder, of radius $R>\frac{d \sqrt{n-1}}{\pi}$, with height function $\rho_{0}$ such that the solution, $\rho(t)$, to the flow (2.1) with initial condition $\rho_{0}$ exists for all time and converges to zero. Then there exists a neighborhood, $O$, of $\rho_{0}$ in $h_{\frac{\partial}{\partial z}}^{1+\beta}\left(\mathscr{C}_{R, d}^{n}\right), 0<\beta<1$, such that for every $v_{0} \in O$ the solution to (2.1) with initial condition $v_{0}$ exists for all time and converges to a function near zero whose graph is a cylinder.

Proof. This follows by the same arguments given in [9]. Set $V \subset h_{\frac{\partial}{\partial z}}^{1+\beta}\left(\mathscr{C}_{R, d}^{n}\right)$ to be the neighborhood of zero given in Theorem 1.1. Since $\rho(t)$ converges to zero in the $C^{k}$ topology, there exists a time $T$ such that $\rho(T) \in V$ and as $V$ is open there exists an open ball $B_{\epsilon}(\rho(T)) \subset V$ of radius $\epsilon$ centered at $\rho(T)$. By the finite time continuity of the volume preserving mean curvature flow there exists a ball $B_{\delta}\left(\rho_{0}\right)$ such that if $v_{0} \in B_{\delta}\left(\rho_{0}\right)$ then the solution, $v(t)$, to (2.1) with initial condition $v_{0}$ exists for $t \in[0, T]$ and $v(T) \in B_{\epsilon}(\rho(T))$. Since $v(T)$ is in $V$, by Theorem 1.1 the solution to (2.1) with initial condition $v(T)$ converges to a function near zero that defines a cylinder. By uniqueness of the flow we get the result.

\section{References}

[1] H. Amann, Nonhomogeneous linear and quasilinear elliptic and parabolic boundary value problems, in Function spaces, differential operators and nonlinear analysis (Friedrichroda, 1992), Teubner-Texte Math., 133, Teubner, Stuttgart, 1993, 9-126.

[2] H. Amann, Linear and quasilinear parabolic problems. Vol. I, vol. 89 of Monographs in Mathematics 89, Birkhäuser Boston Inc., Boston, MA, 1995, Abstract linear theory. 
[3] M. Athanassenas, Volume-preserving mean curvature flow of rotationally symmetric surfaces, Comment. Math. Helv. 72(1) (1997), 52-66.

[4] A.L. Besse, Einstein manifolds, in Classics in Mathematics, SpringerVerlag, Berlin, 2008, reprint of the 1987 edition.

[5] J. Bode, Mean curvature flow of cylindrical graphs, $\mathrm{PhD}$ thesis, Max-Planck Institute for Gravitational Physics (Albert-Einstein Institute) and The Free University of Berlin, 2007.

[6] J. Escher and G. Simonett, Classical solutions for Hele-Shaw models with surface tension, Adv. Differ. Eq. 2(4) (1997), 619-642.

[7] J. Escher and G. Simonett, A center manifold analysis for the MullinsSekerka model, J. Differ. Eq. 143(2) (1998), 267-292.

[8] J. Escher and G. Simonett, The volume preserving mean curvature flow near spheres, Proc. Amer. Math. Soc. 126(9) (1998), 2789-2796.

[9] C. Guenther, J. Isenberg and D. Knopf, Stability of the Ricci flow at Ricci-flat metrics, Commun. Anal. Geom. 10(4) (2002), 741-777.

[10] G. Huisken, The volume preserving mean curvature flow, J. Reine Angew. Math. 382 (1987), 35-48.

[11] H. Li, The volume-preserving mean curvature flow in Euclidean space, Pacific J. Math. 243(2) (2009), 331-355.

[12] A. Lunardi, Analytic semigroups and optimal regularity in parabolic problems, in Progress in Nonlinear Differential Equations and their Applications, 16, Birkhäuser Verlag, Basel, 1995.

[13] G. Simonett, Center manifolds for quasilinear reaction-diffusion systems, Differ. Integral Equ. 8(4) (1995), 753-796.

[14] A. Vanderbauwhede and G. Iooss, Center manifold theory in infinite dimensions. In Dynamics reported: expositions in dynamical systems, $\mathbf{1}$, Dynam. Report. Expositions Dynam. Systems (N.S.), Springer, Berlin, 1992, 125-163. 
Motion by volume preserving mean curvature flow near cylinders 889

Monash University

School of Mathematical Sciences

Monash University VIC 3800

Australia

E-mail address: david.hartley@monash.edu

Received September 29, 2012 
